\title{
The “Motion-Blind”' Patient: Low-Level Spatial and Temporal Filters
}

\author{
R. H. Hess, ${ }^{1}$ C. L. Baker, Jr., ${ }^{2}$ and J. Zihl ${ }^{3}$ \\ 'Physiological Laboratory, University of Cambridge, Cambridge CB2 3EG, United Kingdom, 'Department of Psychology, \\ McGill University, Montreal H3A 1B1, Canada, and ${ }^{3}$ Max-Planck Institute for Psychiatry, D8000 Munich 40, Federal \\ Republic of Germany
}

The "motion-blind" patient previously described by Zihl et al. (1983) was investigated using standard psychophysical procedures with stimuli whose spatial and temporal properties could be separately manipulated. Detection experiments for sinewave grating stimuli of varying spatial and temporal frequency showed sensitivity in this patient to be only slightly impaired. Temporal integration for stimuli of varying spatiotemporal frequency exhibited the expected space-time covariation seen in normal vision.

An examination of the suprathreshold discriminative capacity of this patient was undertaken for spatial frequency, contrast, and temporal frequency. Although all of these discriminative functions were impaired, those concerning temporal frequency or velocity were dramatically reduced. No similar loss was seen for spatial frequency discrimination for moving or temporally varying stimuli. No measurable temporal frequency discrimination was present above $6 \mathrm{~Hz}$ and no velocity discrimination above $6 \%$ sec. Experiments involving the direction discrimination of suprathreshold drifting gratings of arrays of random dots revealed an inability to perceive direction of movement above a velocity of about $6 \% / \mathrm{sec}$. Contrast thresholds contingent on direction of motion of a drifting grating also showed a much greater deficit than simple detection. Apparent motion using 2-flash random dot kinematograms revealed that the residual motion vision of this patient corresponded to the "short-range" motion process of normal vision. This process originally defined by Braddick (1974) operates over restricted space and time intervals. Apparent motion could only be supported by a narrow range of intermediate spatial displacements. These results suggest that this patient does exhibit some residual motion perception, probably corresponding to a severely impaired "short-range" mechanism. The patient's relatively intact ability to perform simple types of discrimination but severe impairment of performance at making judgments relevant to the nature of motion of the same stimuli suggests that while the components necessary for the analysis of motion are intact their more global associations have been

\footnotetext{
Received June 21, 1988; revised Sept. 6, 1988; accepted Sept. 13, 1988.

We gratefully acknowledge a Twinning $(86 / 38)$ grant from the European Science Foundation and support from the Wellcome Trust, Medical Research Council $(8421298 / \mathrm{N}$ ) and a Canadian NSERC grant (A1978) to C.L.B. R.F.II. is a Wellcome Senior Lecturer. We are indebted to Christel Schmidt and to our patient for the countless hours that they spent in our psychophysiological experiments.

Correspondence should be addressed to R. F. Hess, University of Cambridge, Physiological Laboratory, Downing Street, Cambridge CB2 3EG, England.

Copyright (C) 1989 Society for Neuroscience $0270-6474 / 89 / 051628-13 \$ 02.00 / 0$
}

disrupted. This implicates an extrastriate locus of the brain damage. Alternative explanations for the nature of the deficit are discussed.

In 1983, Zihl et al. reported findings on a patient who had suffered bilateral damage to a circumscribed area of the posterior temporal cortex as a result of a thrombosis. All visual functions were found to be intact except that the patient complained of severe difficulties with objects in motion. The following visual functions were reported to be within normal limits: visual acuity, stereopsis, color discrimination, temporal resolution, dynamic and static perimetric fields, and saccadic localization. In contrast to this, motion perception was found to be impaired in the following ways. First, no clear perception of motion was experienced above velocities of $14 \% \mathrm{sec}$ and only an impoverished perception below that. Second, direction discrimination was never greater than $85 \%$ correct even for velocities as low as $2 \%$ sec. Third, velocity discrimination was grossly impaired above $10 \% \mathrm{sec}$. These findings when taken at face value suggest a deficit that is selective for visual motion.

While the exact cytoarchitectural equivalence of cortical regions in monkey and man is not presently known, there are areas within this part of the prestriate cortex in monkey that are known to be involved in the processing of visual motion. Single-cell recording in normal monkey has demonstrated that neurons in the medial temporal area of the superior temporal sulcus [originally identified by Zeki $(1971,1974)$ and termed V5; also referred to as MT] are especially responsive to moving stimuli.

Neurons in area V 5 or MT have been shown to exhibit strong directional selectivity and velocity tuning (Dubner and Zeki, 1971; Zeki, 1971, 1974; Maunsell and van Essen, 1983; Mikami et al., 1986a, b; Newsome et al., 1986). These neurons differ from their counterparts in V1 in 3 important ways. First, they rcspond to the velocity of a moving stimulus rather than its spatial and temporal components (J. A. Movshon, personal communication). Second, they exhibit motion opponency (Allman et al., 1985), and third, on average, area V5 (MT) neurons have larger receptive fields and respond to higher velocities than do those in V1 (Zeki, 1974; Mikami et al., 1986a, b). Additional evidence for the role of area V5 (MT) in the processing of visual motion comes from the behavioral deficits that occur as a consequence of discrete lesions within this area. Newsome et al. (1985) report topographically related deficits in saccadic and pursuit eye movements contingent on moving stimuli as a result of discrete lesions to V5 or MT. Andersen and Siegel (1987) report a selective disturbance in the perception of structurefrom-motion after similar lesions to this area. 
The possibility that this "motion blind" patient may have a lesion that selectively affects a cortical region corresponding to area V5 (MT) in monkey was recognized by Zihl et al. (1983). If so, a careful study of the properties of the perceptual deficit might tell us something about the role played by this cortical area in man, and eventually lead to closer links between human psychophysics and monkey neurophysiology. Although the original report by Zihl et al. was extensive in its assessment of general visual functions, it did not set out to investigate the spatial and temporal properties of the motion deficit. Such measurements might indicate whether the cortical involvement is more extensive, including, for example V1. As the first step we set out to investigate whether the deficit in motion perception is a secondary consequence of some elementary and profound impairment of the spatial and/or temporal processing rather than a deficit specific to motion per se. The only information available from the initial study (Zihl et al., 1983) on the integrity of these functions is that spatial and temporal resolution were found to be normal. It seems appropriate therefore to ask whether the sensitivity of the visual neurons with filtering properties corresponding to lower space and time frequencies are also normal since this is the range that subserves normal motion perception. In doing this we are assessing whether a lesion peripheral to the prestriate cortex could explain the deficit.

In this paper we extend the investigation using a variety of standard psychophysical procedures for stimuli whose spatial and temporal properties could be manipulated separately, and we address 3 questions on which the interpretation of this perccptual deficit depends. (1) Are the peripheral (i.e., to V5) spatial and temporal filters of normal sensitivity and organization? (2) Can their outputs be accurately compared? (3) Is the motion deficit selective for velocity or for temporal frequency?

The results suggest that the peripheral spatial and temporal filters are of approximately normal sensitivity and organization and that the output of the spatial filters can be accurately compared for nontemporal tasks. The deficit is selective for temporal discrimination tasks and in particular those involving motion. The residual motion perception of this patient is best described in terms of temporal frequency rather than velocity, and appears to represent residual function of the short-range process.

\section{Materials and Methods}

\section{General details concerning the patient}

In the experiments described we sought to tailor our experimental procedures to reveal the patient's optimal performance while minimizing the influence of any nonspecific effect of brain damage. In particular we sought whenever possible to avoid using successive temporal presentations since performance would then be contingent on normal shortterm visual memory. Furthermore, we did not want to restrict the presentation time in case there was some diffuse deficit of attentional processes. We therefore chose to use discrimination tasks involving mainly simultaneous spatial comparisons for stimuli that were present for an indefinite time. The patient was allowed to proceed at her own pace, voluntarily initiating presentation of each trial. Frequent rest periods were allowed, especially when the patient complained of "dizziness" produced by moving stimuli. In all the experiments we supplied a fixation mark and ensured that fixation stability was normal. The patient's responses were abnormally slow. In the case of the discrimination experiments, she would spend tens of seconds viewing the 2 comparison stimuli. However, the results of various experiments were reproducible both on the same occasion and at intervals up to 2 years apart. The cxtent to which the important features of this abnormality could be replicated testifies to both the genuineness of this disorder and to its stability over time.

CT scans were performed in August 1987 and NMR scans in March 1988 (that is 9 and 10 years, respectively, after the brain damage had occurred). There exists a good correlation between the CT and the NMR scans, but, as expected, the NMR scans show the white matter lesions more clearly. Compared with the CT scans shown in Zihl et al. (1983), the location of the bilateral brain damage is nearly the same; it appears, however, also in the CT scan to be larger and more clearly marked. The damage appears to be much more symmetrical than it was in the earlier CT scan. At the low ventricular level, the bilateral damage involves the lateral occipital gyrus, which is more affected on the right side. At the high ventricular level, the lateral occipital gyrus and the middle temporal gyrus are damaged bilaterally. In addition, the posterior portion of the middle and the posterior temporal gyrus (including the posterior superior temporal sulcus) are also affected. At the supraventricular level, the angular gyrus is bilaterally damaged, however, much more so on the right side. The white matter is bilaterally involved at all 3 levels, i.e., the damage affects the temporo-occipital, occipital, temporoparietal, and parieto-occipital white matter.

\section{Subjects}

Normal observers included the authors and laboratory personnel, all of whom have normal visual function without any history of occular or brain pathology. Their vision was $6 / 5$ when corrected, and their visual fields were normal in extent.

\section{General procedures}

In all experiments a 2-alternative forced-choice procedure was used. For the detection experiments this involved temporally successive comparisons, whereas for the discrimination experiments it involved spatially simultaneous comparisons. All grating stimuli were generated with a PDP 11/34A laboratory computer connected via a CED 502 interface to either a Joyce screen (for grating experiments) or a Tektronic $x-y$ display (for random dot experiments). Contrast was varied with digital attenuators operating in $0.25 \mathrm{~dB}$ steps. To provide the much finer steps needed for contrast discrimination, 2 such attenuators were used in parallel, one providing the pedestal and the other the increment. The Joyce screen had a $\mathrm{P} 4$ phosphor, $100 \mathrm{~cd} / \mathrm{m}^{2}$ mean luminance, $200 \mathrm{~Hz}$ frame rate, and a $20 \times 30 \mathrm{~cm}$ display area. The Tektronics display had a P36 phosphor and a $20 \times 20 \mathrm{~cm}$ usable display arca. The paticnt viewed the stimuli binocularly with natural pupils, usually from 2.28 meters (except for the high spatial frequency contrast measurements, for which the viewing distance was 4.56 meters).

\section{Specific procedures}

Contrast thresholds. All contrast thresholds were measured using a 2-temporal-alternative forced-choice procedure. The stimuli were sinusoidal in space and time and presented within a Gaussian time envelope of sigma $500 \mathrm{msec}$ (except where this was the variable). The field size was $5^{\circ} \times 7.5^{\circ}$ for spatial frequencies up to $10 \mathrm{c} / \mathrm{d}$, after which it varied inversely with viewing distance. A standard interleaved staircase procedure was used to obtain the $75 \%$ correct point on the psychometric function. The step size was $0.5 \mathrm{~dB}$, and 10 reversals were averaged to obtain an estimate of the mean and SE of the threshold. Further details of this procedure are given by Hess and Pointer (1985).

Suprathreshold discriminations. All discriminations were measured with the 2 comparison stimuli simultaneously present in a bipartite field with unlimited viewing time. Usually 40 and sometimes 80 trials were presented with stimulus conditions randomly interleaved. The results were fitted by cumulative Gaussian distributions using probit analysis to obtain the $75 \%$ correct threshold. The contrast of these stimuli was randomly varied from trial to trial over a $\pm 6 \mathrm{~dB}$ range to ensure that perceived contrast could not be used as a secondary cue to aid spatial and temporal discriminations. Spatial phase relative to the Gaussian envelope was also randomized to ensure that this was not used as a secondary cue in the spatial discriminations.

Random dot apparent motion. These stimuli were similar to those described by Baker and Braddick (1985). Each display consisted of 2 arrays of randomly positioned dots in a $10^{\circ} \times 10^{\circ}$ field, presented in succession on a computer-controlled $x-y$ display oscilloscope. Fach frame was presented for $500 \mathrm{msec}$, with an interframe interval of 5-20 msec (determined by the refresh rate), giving a total stimulus duration of 1000 msec. Each frame consisted of an $N \times N$ matrix of pixel locations, each of which was either empty or contained a dot. Dots occurred in each pixel location with $50 \%$ probability. The second matrix was identical to the first, except for a uniform displacement of all the dots. The displacement was an integral number of pixels; a conventional "wrap- 
around" scheme was used, in which dots displaced beyond a matrix boundary reappeared on the opposite side. Viewing distance was 1 meter.

The spread of each individual dot on the screen was such that individual dots were just visibly distinct when they were spaced at $0.5 \mathrm{~mm}$ (the pixel spacing in the experiments described was always at least twice this). A packed array of dots at this spacing had a luminance of $88 \mathrm{~cd} /$ $\mathrm{m}^{2}$, which can thus be taken as an appropriate estimate of the luminance of a dot. The dots appeared on a background luminance of $34 \mathrm{~cd} / \mathrm{m}^{2}$ from room illumination on the screcn. Other aspects of the display are described more fully by Baker and Braddick (1985).

The subject's task was to discriminate the direction of motion (left or right) of the uniformly displaced dots. The orientation of the displacement was held constant. Thresholds were obtained using a method of constant stimuli with 20-60 trials per condition. Trials were preceded by a tixation mark, whose location indicated the center of subsequent displays. The subject initiated each trial by a button press. All of the conditions being tested in each experiment were randomly interleaved.

"Drifting" random dots. These stimuli consisted of an array of randomly positioned dots, whose horizontal locations were incremented on successive frames. The appearance was of smooth continuous drift and so we refer to them as "drifting" random dots. Dots drifting beyond the pattern border were "wrapped around" to the opposite side. In other respects this stimulus was like that described above. A similar direction discrimination task was employed, using a method of constant stimuli to measure the percentage error as a function of drift velocity. The presentation time was $1 \mathrm{sec}$.

\section{Results}

\section{Detection: sensitivity of peripheral filters}

We began by investigating whether the deficit in motion perception was secondary to some elementary and profound impairment of the spatial and/or temporal processing rather than a deficit specific to motion per se.

The measurement of spatial and temporal contrast sensitivity functions provides an assessment of the overall sensitivity of the peripheral filters (i.e., neurons more peripheral to V5) presumed to be necessary for motion perception. The results of such measurements are displayed in Figure 1. In this, as well as the following figures, the sensitivity of a normal observer (open symbols) is compared with that of the motion-blind patient. The spatial contrast sensitivity for stationary gratings is displayed in Figure $1 A$, the temporal contrast sensitivity for a $1 \mathrm{c} /$ deg contrast reversing grating is displayed in $1 B$, and similar measurements for a drifting grating stimulus are displayed in $1 C$. In each case, the solid curve is the best-fitting (by eye) curve to the normal data, and it has been displaced vertically by about a factor of 2 so that it can be compared with the patient's data. Spatial and temporal sensitivity functions for the motion-blind patient are of the normal shape but are reduced in sensitivity to a relatively small extent. Similar results were found for temporally varying stimuli, whether drifting or contrast-reversing (compare Fig. 1, B, C). These results for the patient fall just outside the expected $95 \%$ confidence limits for a normal population.

Although psychomctric functions for this task were not measured, the accuracy of our patient's thresholds (i.e., the SD from the staircase procedure) was indistinguishable from that of a normal subject, so we would expect the slopes of her psychometric functions to be reasonably normal.

As a further index of the relative functional integrity of the peripheral filters in this patient, we measured temporal integration for a range of spatiotemporal stimuli. We were particularly interested in whether one could demonstrate the covariation in the spatial and temporal properties of the underlying filters response known to exist for normal vision. In normal vision, the mechanisms responding to low spatial frequencies exhibit a more transient response than those tuned to higher spatial frequencies. This is reflected in shorter integration time and a loss of sensitivity at long integration times (Rubson, 1966; Tolhurst, 1975; Watson and Nachmias, 1977; Burr, 1979).

To address this question, we measured contrast sensitivity as a function of stimulus exposure time (SD of the Gaussian temporal envelope). The results are displayed for stationary stimuli in Figure 2 and for drifting stimuli in Figure 3. In each figure, results for the normal observer (open symbols) are compared with those of the motion-blind patient. The solid curve is the best fit (by eye) to the normal data and has been displaced vertically so that it can be compared with the patient's data. In all cases, temporal integration was found to be of a normal form in the motion-blind patient. In agreement with the previous contrast sensitivity measurements, the patient's overall sensitivity was slightly reduced at all exposure durations (about a factor of 2). Furthermore, the patient's results showed the more "transient" behavior at low, as compared with high, spatial frequencies that is seen in normal subjects (compare Fig. 2, $A$, $B)$.

\section{Discrimination: cross-comparison of peripheral filters}

The extraction of velocity information, so important for the processing of visual motion, requires that peripheral spatial and temporal filters be of approximately normal sensitivity and that their outputs be accessible for accurate comparison. The above data suggest that in this patient the first of these requirements is fulfilled. In assessing the second we tried to explore a more general explanation for the "motion-blindness" in this patient, namely, that there is a general disability for tasks that critically depend on cross-filter comparisons, with a consequent impact on motion perception.

To assess this proposal we compared discrimination performance relying on cross-filter comparisons for a series of visual tasks: contrast and spatial frequency discrimination, and temporal frequency and drift rate discrimination. In all cases, the method of constant stimuli (usually 40 trials per condition) was used in conjunction with a simultaneous spatial 2-alternative forced-choice (2AFC) paradigm. The exposure duration was unlimited. We chose this paradigm so that we would not need to make any additional assumptions about the patient's shortterm memory (important for temporal $2 \mathrm{AFC}$ ) and focal attention (important for limited exposure durations).

Figure 4 displays the results for contrast discrimination at pedestal contrasts of $3 \%(A), 10 \%(B)$, and $30 \%(C)$ for a normal (open symbols) subject and for the motion-blind patient. The stimulus was a $1 \mathrm{c} / \mathrm{deg}$ stationary sinewave grating. The percentage correct in the $2 \mathrm{AFC}$ task is plotted against the contrast of the increment. Here and in all subsequent figures in this section the increment is expressed as a percentage of the base frequency and plotted logarithmically on the abscissa. This facilitates comparison of relative slopes of psychometric functions when performance is impaired. The solid curves represent best fits using probit analysis. For all pedestal contrasts (the stimulus was a $1 \mathrm{c} / \mathrm{deg}$ stationary sinewave grating), the patient's performance was consistently reduced by about a factor of 3 . However, the psychometric slopes are not statistically different from normal. Similar results for spatial frequency discrimination are seen in Figure 5, where discriminations at 3 stationary base spatial frequencies $(0.25 \mathrm{c} / \operatorname{deg}$ in $A ; 1 \mathrm{c} / \operatorname{deg}$ in $B ; 10.5 \mathrm{c} / \operatorname{deg}$ in $C)$ are compared. In this and subsequent figures of this section, 

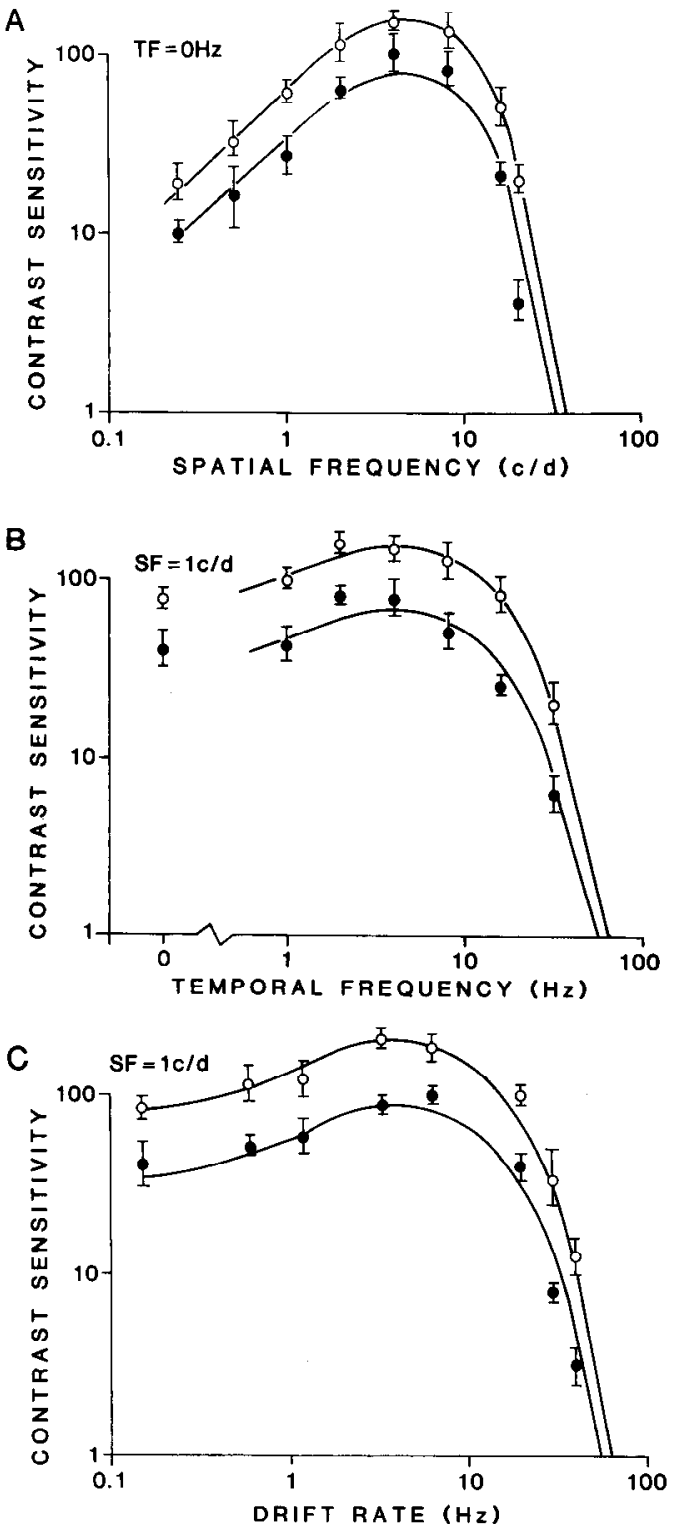

Figure 1. Contrast sensitivity plotted against spatial frequency $(A)$, temporal frequency $(B)$, and drift rate $(C)$ for a normal observer (open symbols) and the motion-blind patient (filled symbols). In $A$, the stimulus was a stationary sinewave grating; in $B$, it was a sinewave grating, sinusoidally contrast-reversing in time; in $C$, it was a drifting sinewave grating. Means are displayed \pm 1 SE. In each case, the curve that best fits the normal results also provides a good fit to the patient's results when displaced vertically.

the stimuli were always sinewave gratings contained within a Gaussian window in the horizontal dimension. The contrast with respect to the window was randomized from trial to trial over a range of \pm a factor of 2 . The spatial phase of the stimuli with respect to the window was also randomized from trial to trial. This ensured that the subsidiary cues of relative phase and perceived contrast did not aid the discrimination.

For the lowest spatial frequency the bright bars appeared wider than the dark bars. This is a well-known perceptual, rather than physical, distortion. The 2 curves on the right of Figure $5 A$ represent discriminations based on attending to either the bright bars $(\mathrm{X})$ or the dark bars. The spatial frequency discrimination results of Figure 5 suggest that the patient's performance
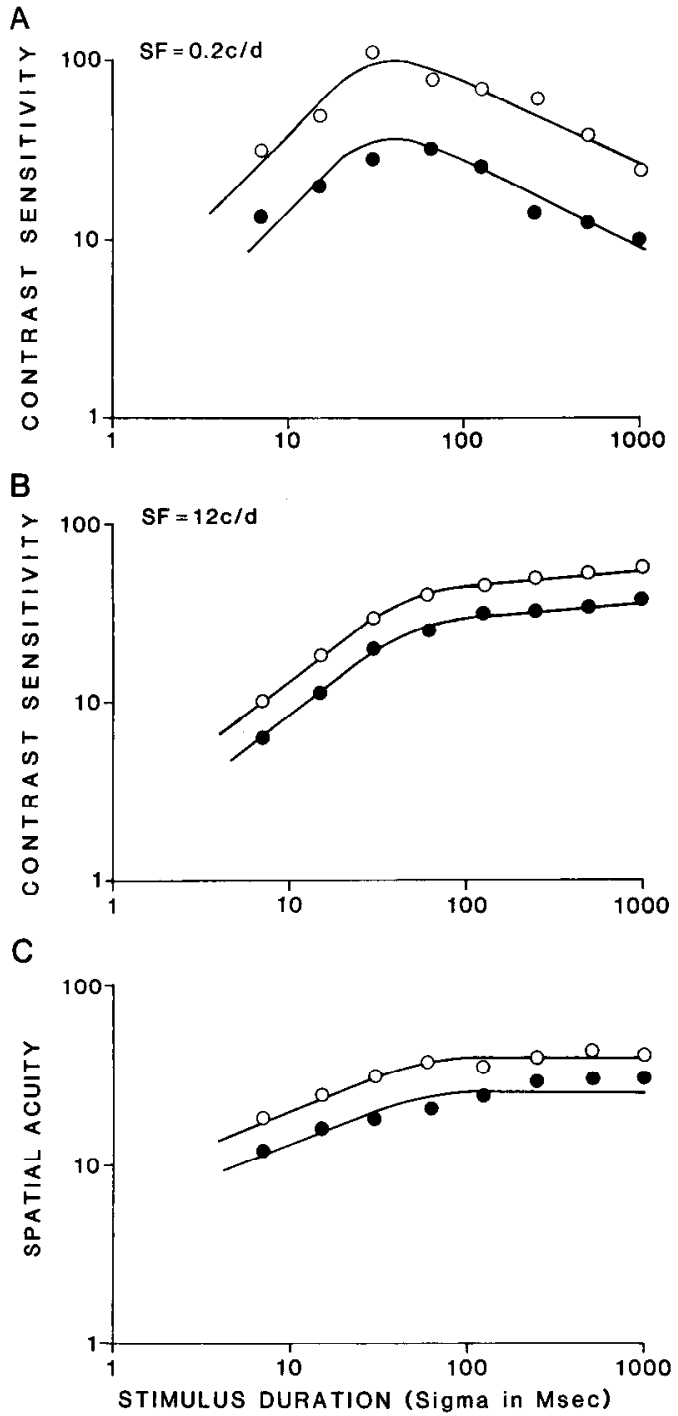

Figure 2. Contrast sensitivity plotted against exposure duration for 2 stationary sinewave gratings, namely, a $0.2 \mathrm{c} / \mathrm{deg}(A)$ and a $12 \mathrm{c} / \mathrm{deg}(B)$ grating. Note that in $C$, spatial acuity is plotted against exposure duration. In $C$, the spatial acuity for the normal subject under our experimental conditions was $39 \mathrm{c} / \mathrm{deg}$, whereas for the patient it was $28 \mathrm{c} / \mathrm{deg}$. The curve that best fits the normal subject's data is also used to fit the patient's data. In all frames, results are compared for the patient ( filled symbols) and a normal observer (open symbols). Stimuli were presented within a Gaussian time envelope, and sigma refers to the time over which this Gaussian falls to $1 / e$ of its peak value on one side.

is reduccd at all spatial scalcs by about a factor of 5 . Again the slopes of the psychometric functions are similar to normal.

Results for a similar experiment but involving temporal frequency discrimination are displayed in Figure 6 for 3 base temporal frequencies $(0.15 \mathrm{~Hz}$ in $A ; 0.5 \mathrm{~Hz}$ in $B ; 1 \mathrm{~Hz}$ in $C$ ). The stimuli were $1 \mathrm{c} / \mathrm{deg}$ sinewave gratings sinusoidally contrastreversing in time. Discrimination performance is now a factor of about 10 below normal, but the slopes of the psychometric functions are similar to normal. Discrimination of drift rate was substantially worse (Fig. 7), the deficit being about a factor of 20. In addition, the slopes of some of the patient's psychometric functions appear abnormally shallow.

In a related series of experiments we sought to determine how many discriminable steps could be made by the patient across 

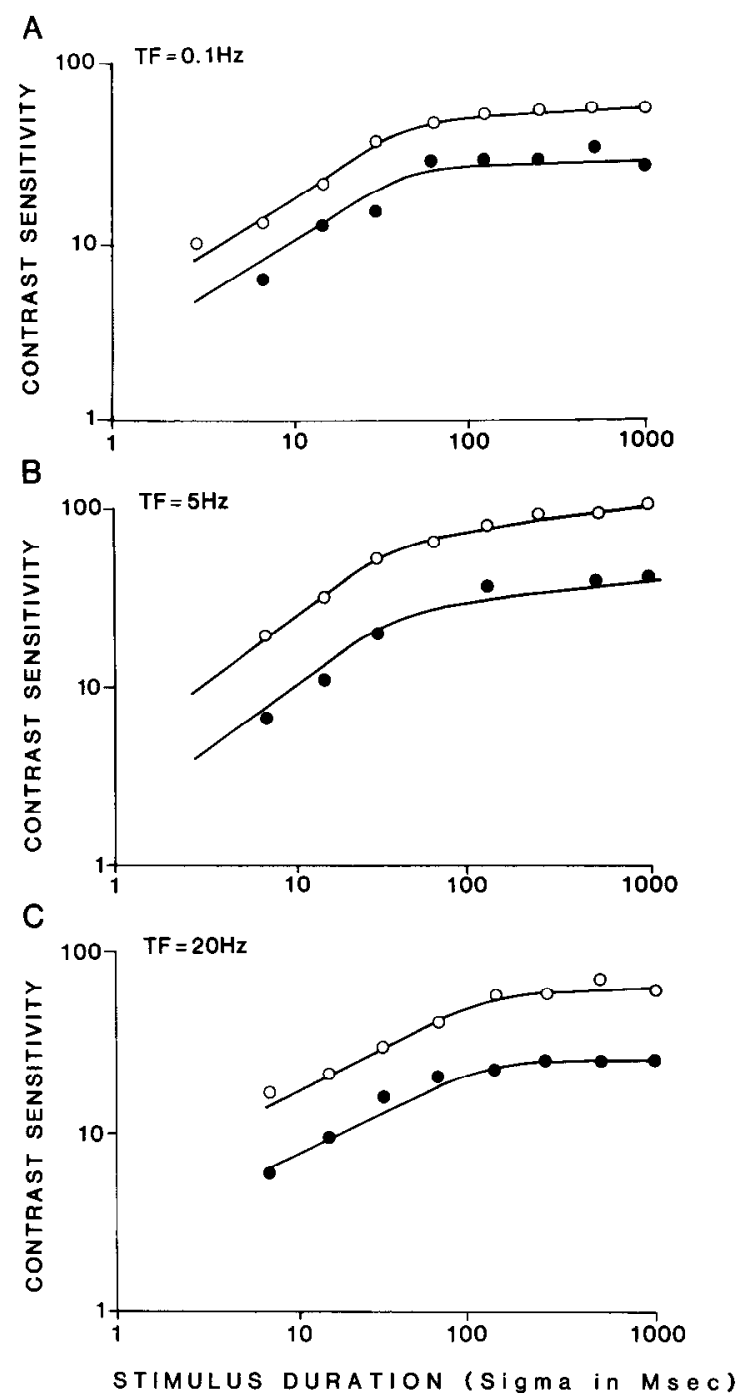

Figure 3. Contrast sensitivity is plotted against exposure duration for a $1 \mathrm{c} / \mathrm{deg}$, sinewave grating drifting at 1 of 3 different rates, namely, 0.1 $\mathrm{Hz}(A), 5 \mathrm{~Hz}(B)$, and $20 \mathrm{~Hz}(C)$. In each panel, the results are compared for the patient and normal observer (filled and open symbols, respectively). The curve that best fits the normal subject's data is also used to fit the patient's data.

a range of temporal frequencies for both contrast-reversing and drifting stimuli. The experimental conditions were otherwise the same as those described above. Results from a normal subject are illustrated schematically by the dashed and dash-dot lines in Figure 8. The abscissa represents the base temporal frequency and the ordinate the test or comparison temporal frequency that can be perfectly discriminated. The logarithmic scales have a discontinuity so as to include " $0 \mathrm{~Hz}$," which refers to a stationary grating with a viewing time of the order of 20 $100 \mathrm{sec}$. For a $1 \mathrm{c} / \mathrm{deg}$ sinewave grating at threshold, $0 \mathrm{~Hz}$ is discriminable from $4 \mathrm{~Hz}$; however, $4 \mathrm{~Hz}$ cannot be discriminated from any higher temporal frequency (Watson and Robson, 1981). This is represented by the dashed lines in Figure 8 showing that only one discriminated step across the entire temporal spectrum is possible at threshold.

For suprathreshold sinewave gratings $(50 \%$ contrast $\pm 6 \mathrm{~dB}$ contrast jitter-see Materials and Methods), the picture is very different and is independent of contrast level as Iong as it is

\section{CONTRAST DISCRIMINATION}
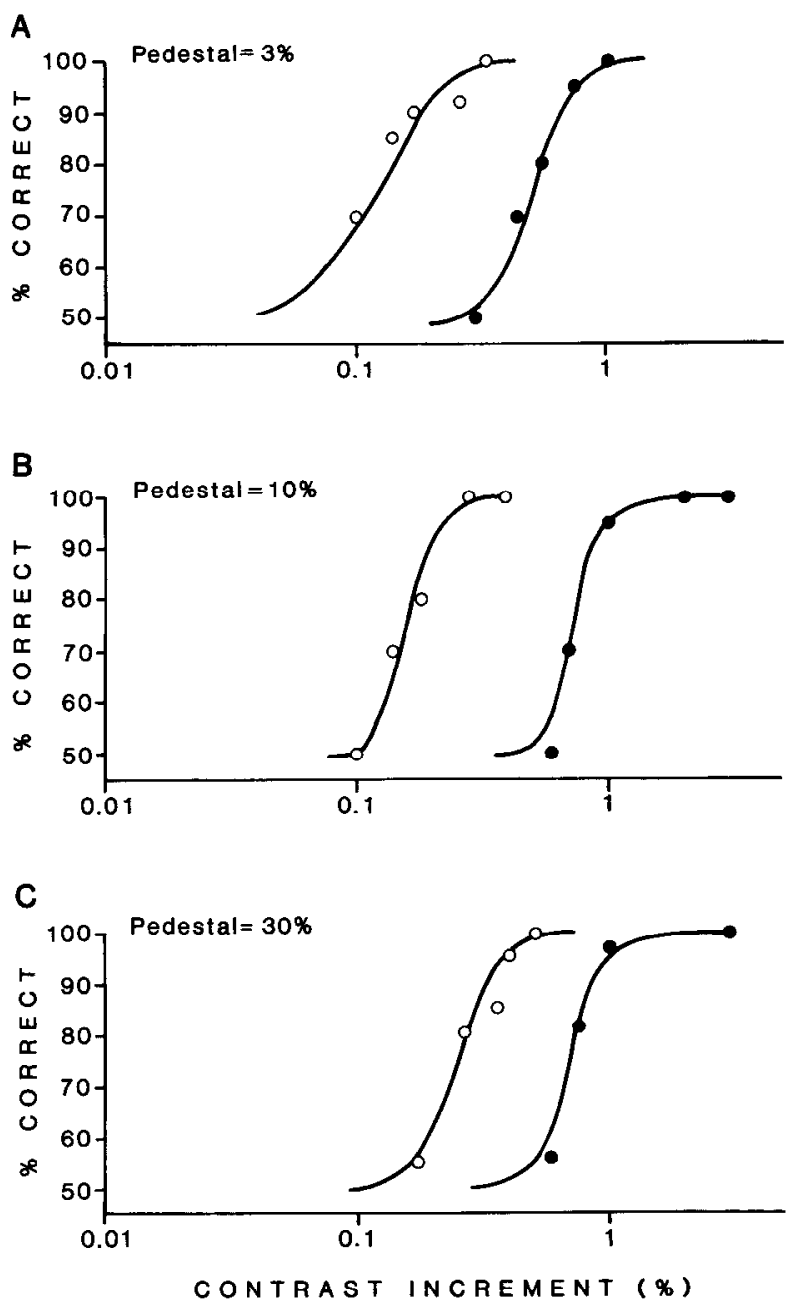

Figure 4. Percent correct response in a 2 spatial alternative contrast discrimination task is plotted against the incremental contrast for the patient (filled symbols) and normal observer (open symbols). Results are shown for 3 different pedestal contrasts, namely, 3\% $(A), 10 \%(B)$, and $30 \%(C)$. These results were obtained from 40 trials and have been fitted by a cumulative Gaussian (solid curves) by Probit analysis to derive $75 \%$ correct threshold values.

more than a factor of 2 above threshold. At low temporal frequencies, the discriminable step size is on the order of $50 \%$ of the base frequency. This declines to around $8 \%$ in the intermediate temporal frequency range $(2-10 \mathrm{~Hz})$ and then increases again in the higher frequency range (Mandler, 1984; Hess and Plant, 1985). This is schematically illustrated by the dash-dot lines in Figure 8 . These 2 limiting cases for normal performance provide benchmarks for the patient's results (solid lines: thin lines for temporal discrimination of contrast-reversal stimuli and thick lines for drift discrimination). For a suprathreshold ( $50 \%$ contrast $\pm 6 \mathrm{~dB}$ contrast jitter) $1 \mathrm{c} / \mathrm{deg}$ temporally modulated sinewave grating, the motion-blind patient could discriminate $0 \mathrm{~Hz}$ from $0.12 \mathrm{~Hz}$ (20-100 sec viewing time), 0.12 $\mathrm{Hz}$ from $1 \mathrm{~Hz}, 1 \mathrm{~Hz}$ from $5 \mathrm{~Hz}$, and $5 \mathrm{~Hz}$ from $20 \mathrm{~Hz}$. A 20 $\mathrm{Hz}$ contrast reversal stimulus could not be discriminated from any higher temporal frequency. Thus, she could make 4 suprathreshold steps in discrimination across the temporal range compared with the 30-40 steps for a normal subject. A similar 


\section{SPATIAL DISCRIMINATION}

A

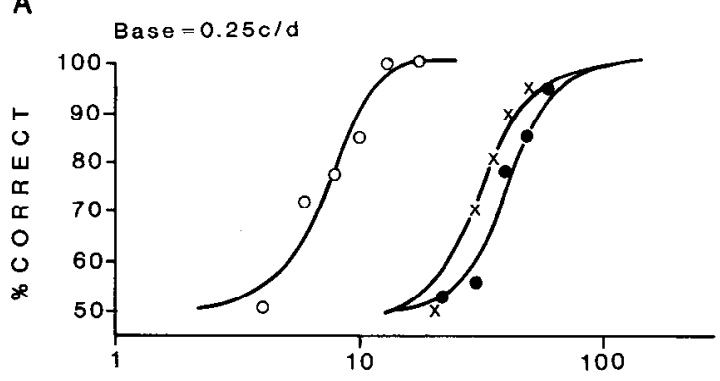

B

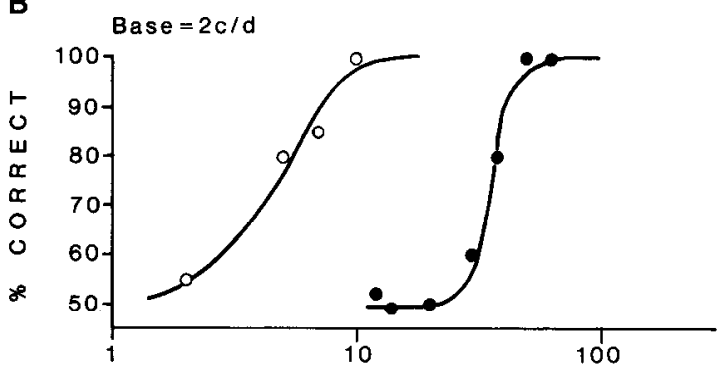

C

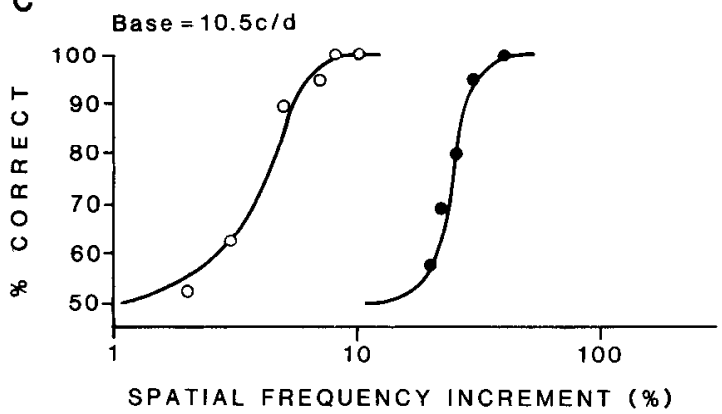

Figure 5. Percent correct response in a 2 spatial alternative spatial frequency discrimination task is plotted against the spatial frequency difference expressed as a percentage of the base spatial frequency. Results are compared with the patient (filled symbols and crosses) and a normal observer at 3 base spatial frequencies: $0.25 \mathrm{c} / \operatorname{deg}(A), 1 \mathrm{c} / \mathrm{deg}(B)$, and $10.5 \mathrm{c} / \mathrm{deg}(C)$. The results have been fitted by a cumulative Gaussian (solid curves) using Probit analysis. At the lowest base spatial frequency $(0.25 \mathrm{c} / \mathrm{deg}$ in $A)$ the light bars appear larger in width than the dark bars. This was also noticeable to the patient, and she was therefore asked to undertake the discrimination task by making judgments of light bars on one run (crosses) and dark bars on another occasion (filled circles).

series of experiments was carried out for drifting stimuli while the patient maintained fixation on the central partition between the 2 spatially separate, drifting stimuli. Now only 3 discriminable steps could be made across the entire temporal range for similar $1 \mathrm{c} / \mathrm{deg}$ suprathreshold gratings. Although $0 \mathrm{IIz}$ could be reliably discriminated from $0.1 \mathrm{~Hz}, 0.1 \mathrm{~Hz}$ from $1 \mathrm{~Hz}$, and $1 \mathrm{~Hz}$. from $6 \mathrm{~Hz}, 6 \mathrm{~Hz}$ could not be reliably discriminated from any higher drift rate. This result was independent of the direction in which the stimuli drifted.

The results of this section when taken together suggest that reduced performance is seen in this patient on tasks involving cross-filter comparisons. For tasks involving stationary stimuli, discrimination performance is reduced by a factor of $3-5$, whereas for tasks involving temporal or velocity discrimination performance is reduced by a factor of 10-20. In the latter case, only
TEMPORAL DISCRIMINATION

A

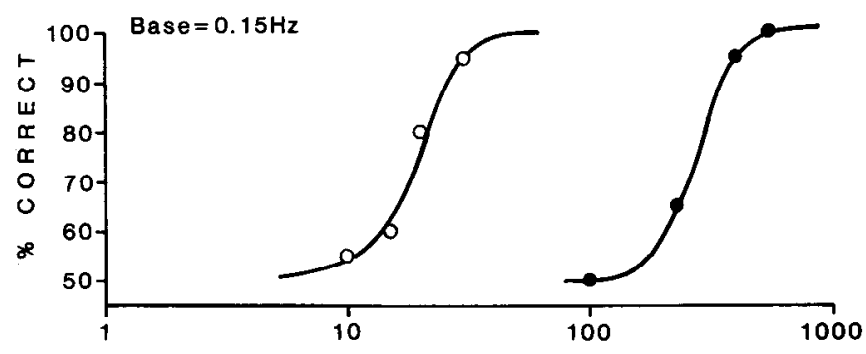

B

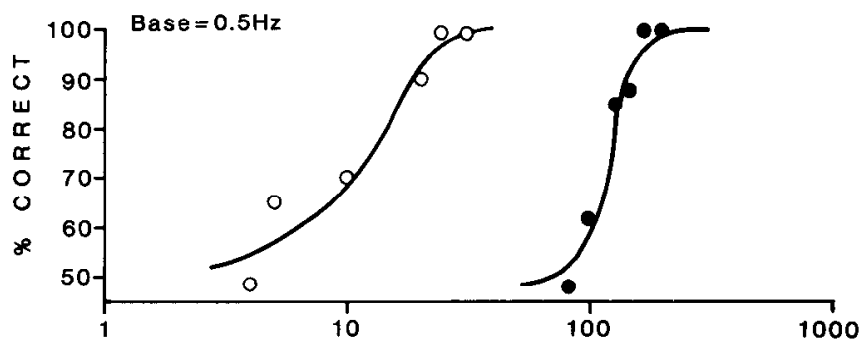

C

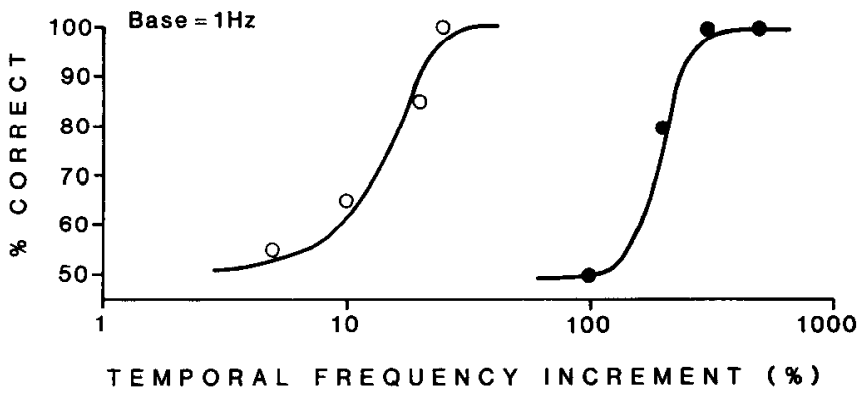

Figure 6. Percent correct response in a 2 spatial alternative temporal frequency discrimination task is plotted against the temporal frequency differences expressed as a percentage of the base temporal frequency (contrast-reversal). Results are compared for the patient (filled symbols) and normal ohserver at three hase temporal frequencies, namely, 0.15 $\mathrm{Hz}(A), 0.5 \mathrm{~Hz}(B)$, and $1 \mathrm{~Hz}(C)$. The solid curves are best-fitting cumulative Gaussian, using Probit analysis.

3-4 discriminable steps are possible across the entire temporal range for suprathreshold stimuli. While this is better than normal threshold performance, it is grossly impaired compared with normal performance above threshold.

The remaining question is whether the greater anomaly seen for temporal and motion discriminations is due to a deficit in the extraction of temporal and motion information or is merely a consequence of a disruptive influence on performance due to temporal modulation. In other words, is this deficit related to the stimulus or to the task? To address this issue, we tested spatial frequency discrimination performance for stimuli that contained temporal modulation. The stimulus was a $1 \mathrm{c} / \mathrm{deg}$ sinewave grating of $50 \%( \pm 6 \mathrm{~dB}$ jitter $)$ contrast, either contrastreversing at $1 \mathrm{~Hz}$ or $10 \mathrm{~Hz}$ or drifting at $1 \mathrm{~Hz}$ or $10 \mathrm{~Hz}$. Results are displayed in Figure 9 for the patient (filled symbols) and for a normal observer (open symbols). The solid curves are those for the comparable experiment where the stimuli to be discriminated were stationary (i.e., those for Fig. 5B). It can be seen 
DRIFT DISCRIMINATION
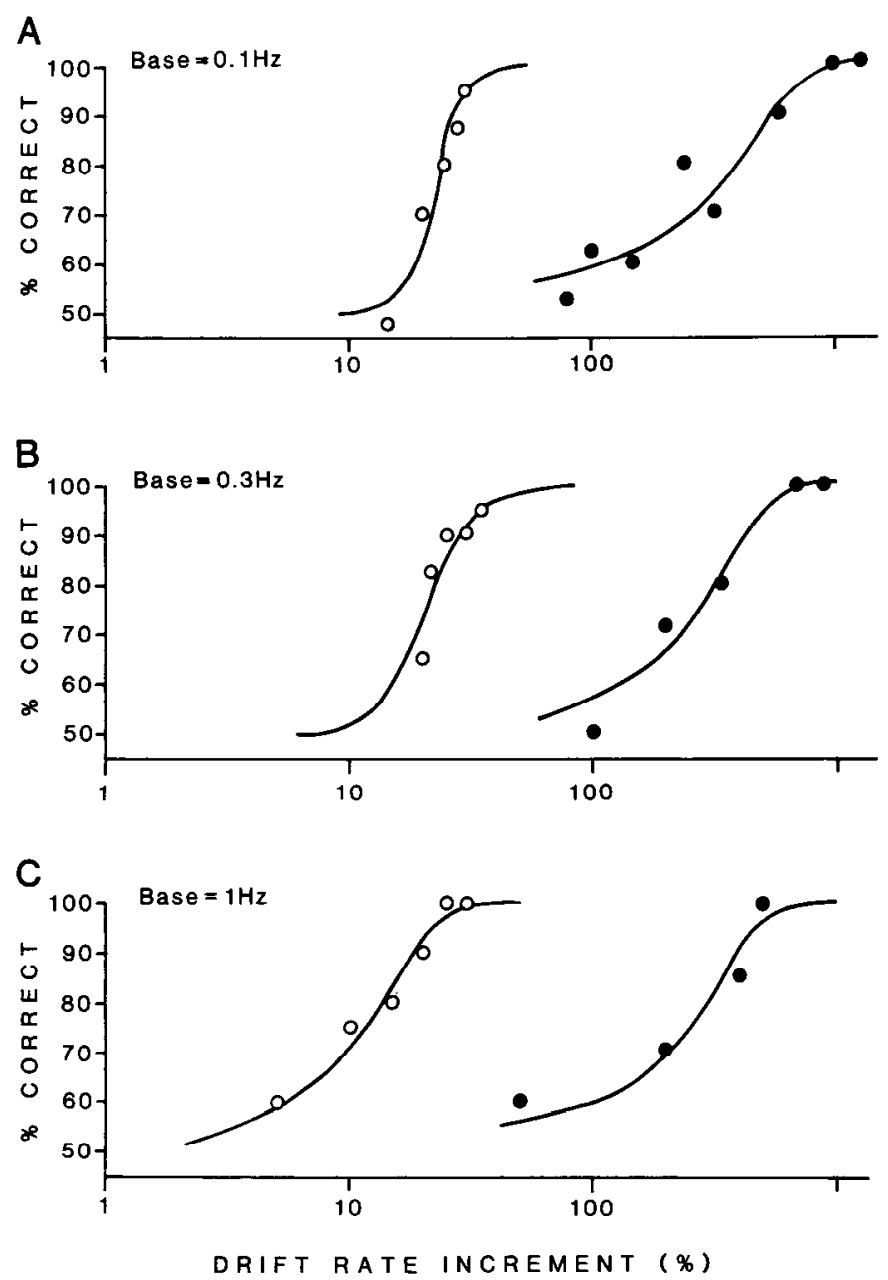

Figure 7. Percent correct response in a 2 spatial alternative drift rate discrimination task is plotted against the drift rate difference expressed as a percentage of the base drift rate. Results are compared for the patient (filled symbols) and normal observer at three base drift rates, namely, $0.1 \mathrm{~Hz}(A), 0.3 \mathrm{~Hz}(B)$, and $1 \mathrm{~Hz}(C)$. The solid curves are bestfitting cumulative Gaussian, using Probit analysis.

that the introduction of temporal modulation into what is an essentially spatial task does not degrade performance (indeed, discrimination was slightly improved in the $1 \mathrm{~Hz}$ drift condition) and performance was much better than for purely temporal discriminations (as in Figs. 6, 7). Thus, the greater abnormality for temporal tasks reported here implies that there is a deficiency in the extraction and comparison of temporal information (including motion), and not just reduced performance for any task involving stimuli that vary temporally.

\section{Direction discrimination: motion mechanisms}

Since the velocity discrimination of this patient is restricted to slow rates of image motion, this leaves open the possibility that it may be derived secondarily from displacement. By this we mean that the subject might track or identify the distance moved per unit time by individual elements of the stimulus as a means of indirectly inferring velocity information. Gratings are not ideal stimuli from this point of view since some sort of relative velocity information could be obtained by, for example, counting how many individual bars disappeared from one edge of the screen per unit time. Such an explanation might appear particularly plausible for the range of velocities for which good performance could be measured in this patient. A stimulus for which a feature-to-feature comparison over time is more difficult is a field of randomly arranged dots which are spatially displaced in an abrupt manner. Such an "apparent motion" stimulus gives a strong impression of smooth motion for sufficiently small spatial displacements and interstimulus intervals (ISI), while making it difficult to discern which dot in the first exposure corresponds to which dot in the second exposure. In other words, any intellectual derivation of direction of motion or velocity, by noting the displacement of individual elements, is considerably more difficult. Another advantage of this stimulus is its analytical value for investigating the spatial and temporal properties of the so-called short-range motion process of normal vision (e.g., Braddick, 1974; Baker and Braddick, 1985). This process operates over limited spatial and temporal spans.

The results of measuring direction discrimination for twoframe apparent motion of random dot stimuli are shown in Figure 10. In Figure $10 A$, the percentage correct for direction discrimination is plotted against the spatial displacement of the dots (see Materials and Methods for details). The exposure duration for each frame was $500 \mathrm{msec}$ and the ISI was $5 \mathrm{msec}$. The normal subject's data are illustrated by open symbols and those of the patient by filled symbols. The patient's performance was never greater than $90 \%$ correct and better-than-chance performance was obtained for a more restricted range of spatial displacements than in a normal subject. The normal limits are typically defined by $D_{\min }$ and $D_{\max }$, the minimum and maximum spatial displacements over which direction discrimination reaches some criterion level (e.g., $75 \%$ correct).

The patient's $D_{\max }$ of $0.37^{\circ}$ was only a factor of about 2 below that of the normal subject's value $\left(0.80^{\circ}\right)$. The patient's $D_{\min }$ value was $0.09^{\circ}$, far more than that of the normal subject $\left(0.007^{\circ}\right)$. However, this type of $D_{\min }$ measure varies a great deal across normal subjects, as seen in Figure 4 of Baker and Braddick (1985); from those data (at a nominal eccentricity of $5^{\circ}$ ), their worst subject exhibitcd a $D_{\min }$ of about $0.03^{\circ}$, only a factor of 3 better than the patient. Of course, this comparison must be somewhat qualified by the different conditions Baker and Braddick (1985) employed, particularly the much longer exposure duration used here, but it would appear that there is a considerable degree of residual function in the patient's "short-range" motion-detection mechanism.

Figure $10 B$ shows the influence of exposure duration, for fixed values of spatial displacement $\left(0.125^{\circ}\right)$ and ISI $(5 \mathrm{msec})$. The patient's performance deteriorated rapidly for exposure durations below $500 \mathrm{msec}$, while normal observers exhibit errorless performance for exposures much less than $100 \mathrm{msec}$ (Baker and Braddick, 1985). Note that this effect does not contaminate the results in Figure $10 \mathrm{~A}$, where the exposure duration was set to 500 msec.

In Figure $10 C$, the effect of ISI is seen for an exposure duration of $500 \mathrm{mscc}$ and a spatial displacement of $0.125^{\circ}$. For normal observers, ISIs above $40 \mathrm{msec}$ degrade performance, this being one of the distinctive properties of the so-called short-range motion mechanism (Braddick, 1974). A similar dependence to that of normal vision is seen in the patient's results, with the exception that completely errorless performance was never reached.

These results argue that the residual motion detection of this patient is not due to the secondary effects of tracking particular 


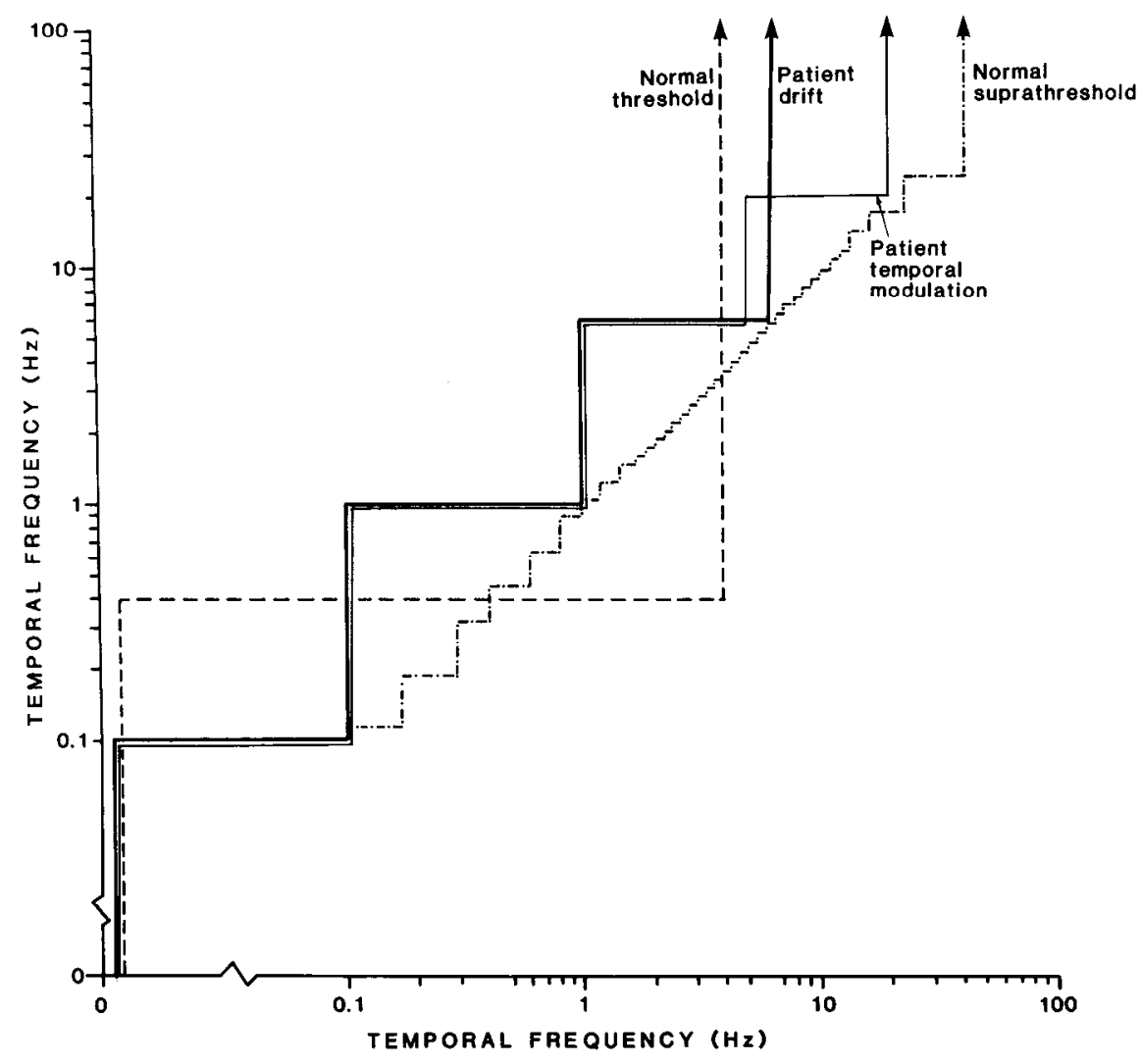

Figure 8. Number and size of the just discriminable ( $75 \%$ correct) steps that can be made across the entire temporal range is illustrated for temporally modulated (contrast-reversing thick solid line) and drifting (thin solid line) sinewave gratings for the patient. These are compared with those for normal suprathreshold vision (dash-and-dot line) and normal threshold vision (dashed line). Each horizontal plateau represents a discriminable step. Note that both scales are discontinuous to accommodate the stationary condition. The normal suprathreshold results are taken from Hess and Plant (1985) and Mandler (1984). stimulus features or by relative displacement judgments. The specificity of the patient's residual motion perception to small spatial displacement and ISIs implicate the short-range process. However, it might still be argued that the mechanisms underlying direction discrimination of the apparent motion of random dot kinematograms and of 1-dimensional gratings are different. In an attempt to test this, we compared the direction discrimination of smoothly moving stimuli, using either arrays of random dots (i.e., a $10^{\circ} \times 10^{\circ}$ array of random dots whose horizontal position was incremented on successive frames) or drifting gratings. The presentation duration was $1 \mathrm{sec}$, the grating contrast was $50 \%$, and its spatial frequency was $1 \mathrm{c} / \mathrm{deg}$. These results are seen in Figure $11 A$ for drifting random dots and in Figure $11 B$ for drifting gratings. In each case, the percentage correct for direction discrimination is plotted against velocity of motion. The results are similar for either stimulus. First, at no velocity did the patient exhibit errorless performance. Second, performance was seriously reduced at very low velocities: below $1 \%$ sec for the random dots and below $0.5 \%$ sec for the grating. Last, performance began to decline at very high velocities: around $8 \% \mathrm{sec}$ for random dots and $4 \% \mathrm{sec}$ for gratings. In this velocity range $\left(0.12-64^{\circ} / \mathrm{sec}\right)$ normal subjects exhibit errorless performance. These similarities in the patient's performance for drifting arrays of random dots and for gratings suggest that the same underlying mechanisms are responsible for the reduced performance and that these mechanisms are signaling motion per se rather than using some secondary cue such as feature tracking or displacement to infer motion.

One remaining question concerning the patient's residual motion perception is whether it is best represented in terms of temporal frequency or velocity. The similarity of the results in Figure $11, A, B$ leave this matter unresolved since grating data are presented at only one spatial frequency. To address this issue we obtained contrast thresholds for direction discrimination (termed direction discrimination thresholds) for drifting gratings at a series of spatial and temporal frequencies. The results are plotted in Figure 12 in terms of temporal contrast sensitivity functions for direction discriminations with the stimulus spatial frequency as a parameter. The normal results are illustrated by open symbols and are very similar to contrast thresholds for detection of the presence of the stimulus (termed simple detec-

\section{SPATIAL DISCRIMINATION}

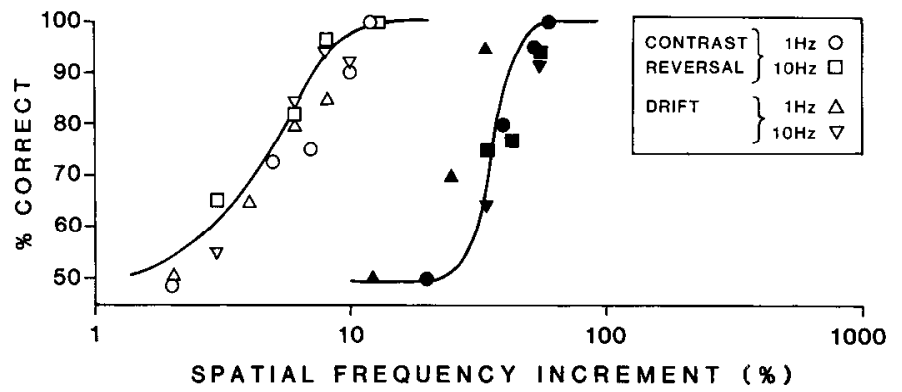

Figure 9. Percent correct response in a 2 spatial alternative spatial frequency discrimination task is plotted against the spatial frequency difference expressed as a percentagc of the base frequency. Results are compared between the patient (filled symbols) and a normal observer for spatial discriminations for stimuli incorporating 2 different temporal factors, namely, temporal modulation (contrast reversal) or drift. Each condition is for 1 and $10 \mathrm{~Hz}$. The solid lines are the best-fitting curves for stationary stimuli derived from the results in Figure 5. Incorporating a temporal modulation or drift does not significantly impair the discriminative function of either the patient or the normal observer. 
A

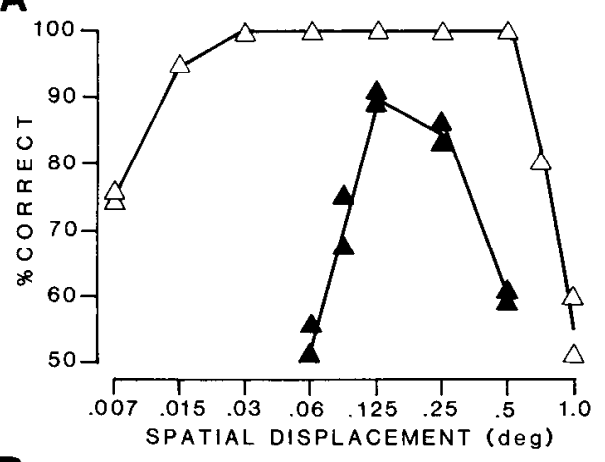

B

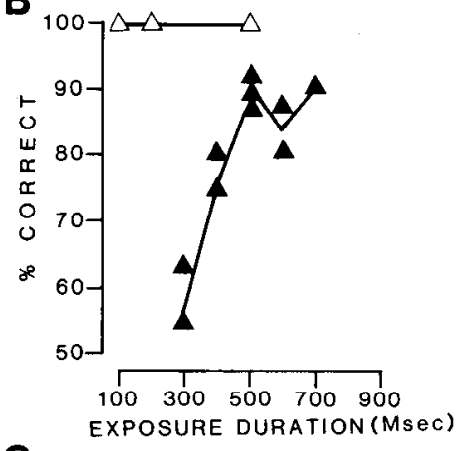

C

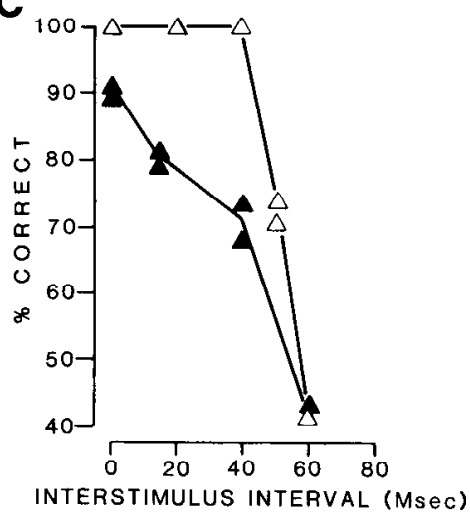

Figure 10. Percent correct response for direction discrimination for apparent motion for 2 flash random dot kinematograms is compared for the patient (filled symbols) and a normal observer. The spatial displacement between each flash presentation which supports apparent motion is seen in $A$, the exposure duration dependency is seen in $B$, and the interstimulus interval dependency is seen in $C$.

tion thresholds). For the patient, the contrast needed to discriminate the direction of motion was grcatly clcvatcd, especially for higher temporal frequencies. In Figure $12 C$ the filled circles represent the contrast necessary for the patient to detect the presence of the drifting stimuli (simple detection) and the filled triangles represent the contrast needed to correctly identify their direction of drift (direction discrimination). A similar pattern was found for drifting gratings of different spatial frequency (compare panels $A-E$ ). Figure $12 F$ shows all these results for different spatial frequencies superimposed. For the normal subject, there was a significant, though small, spatiotemporal interaction and to a first approximation the results can be reasonably well described by a single function. It is clear that the temporal properties of the patient's residual motion perception are independent of spatial frequency, implying that the anomaly
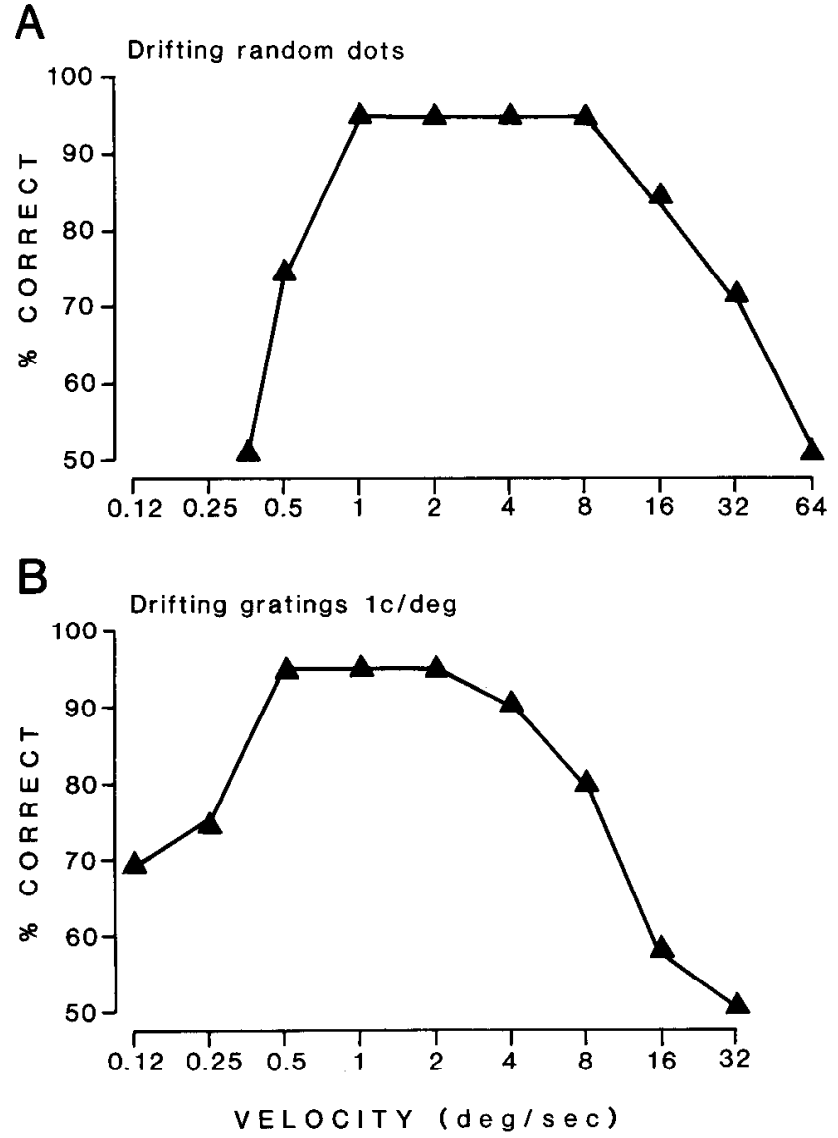

Figure 11. Percent correct response for a direction of motion discrimination task is plotted against the velocity of image motion for arrays of random dots $(A)$ and a drifting sinewave grating of $1 \mathrm{c} / \mathrm{deg}(B)$. The range over which the direction of motion can be discriminated is narrower than normal $(0.12-64 \mathrm{deg} / \mathrm{sec}$ correspond to $100 \%$ correct for normal vision) and even at intermediate velocities where performance is best, it never reaches $100 \%$ correct. The overall performance is comparable for random dots and linear gratings.

is best thought of in terms of temporal frequency rather than velocity.

Figure 13 shows these results replotted in terms of spatial frequency for 2 temporal frequencies. Contrast sensitivity for direction discrimination for the normal subject (open symbols) exhibits a peak around $4 \mathrm{c} / \mathrm{deg}$ with a shallow low spatial frequency roll-off and a steeper high spatial frequency roll-off. The patient's results under comparable conditions (filled symbols) of stimulation have a low-pass characteristic and are somewhat similar in form to that seen in normal vision at higher temporal rates, namely, the spatial sensitivity function found for direction discrimination at $9 \mathrm{~Hz}$ (open circles).

\section{Discussion}

Visual functions unaffected by the lesion

In the original report, Zihl et al. (1983) reported that the following visual functions were within normal limits: visual acuity, perimetric fields, stereopsis, hue vision, and temporal discrimination. In the present study, we retested visual acuity, color discrimination using the FM 100 test, and stereopsis using the TNO test (Julesz sterograms). Visual acuity and hue discrimination were found to be normal as reported in the original paper (Zihl et al., 1983). Stereopsis was slightly impaired but at the 


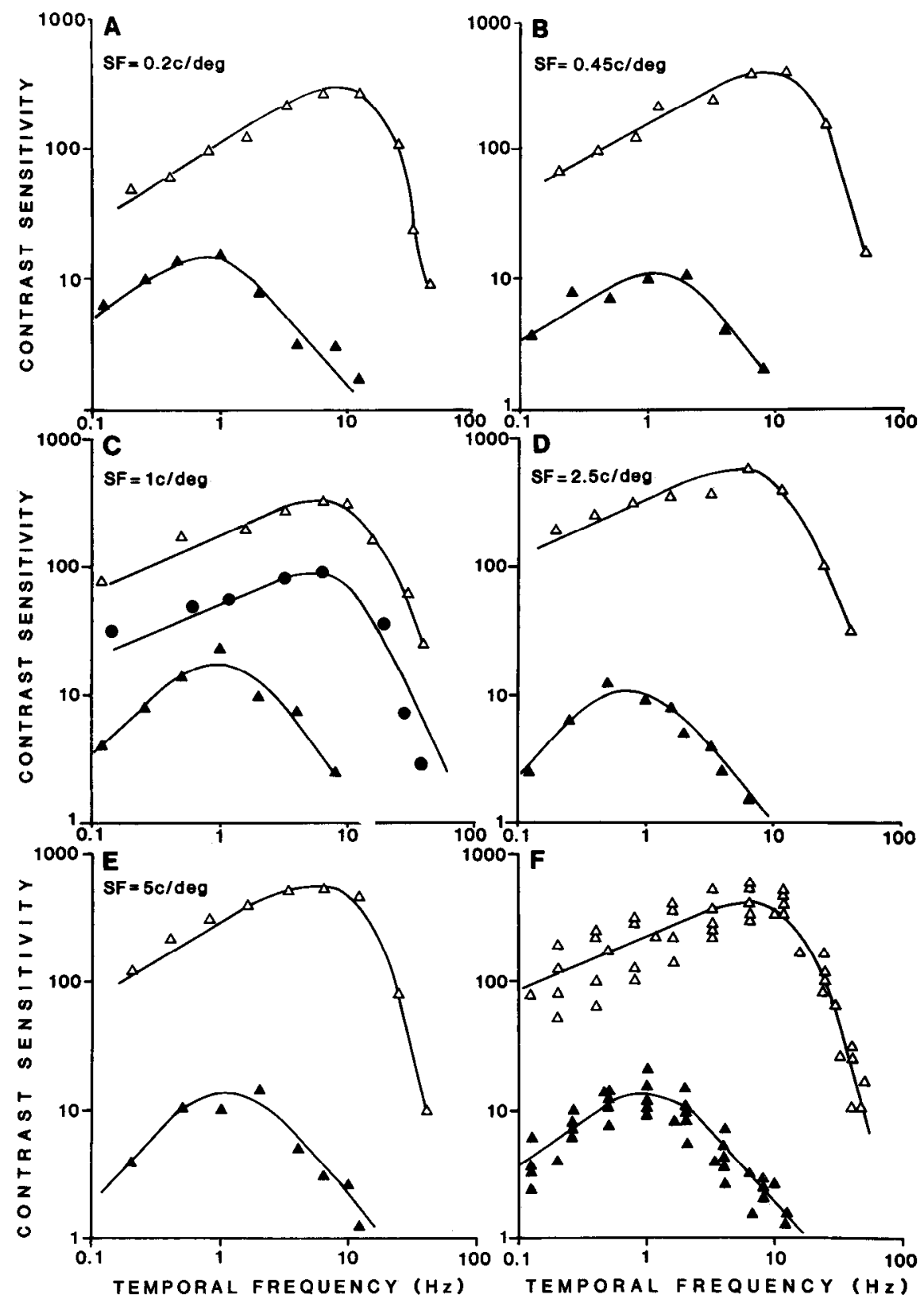

Figure 12. Contrast sensitivity is plotted against temporal frequency for drifting sinewave gratings of random left/right direction. The results of the patient (filled triangles) and normal observer (open triangles) are compared for the correct discrimination of drift direction. Results are shown in frames $A-$ $E$ for spatial frequencies between 0.2 and $5 \mathrm{c} / \mathrm{deg}$. In frame $F$, all of these results are superimposed to show that temporal frequency and not velocity is the appropriate metric. In frame $C$, the filled symbols show the patient's results for the simple detection of the presence of these stimuli. level previously reported (Zihl et al., 1983). Spatial and temporal contrast sensitivity functions are also not markedly abnormal. This is also true to some degree for spatial frequency and contrast discrimination. All of these findings argue for relatively normal function up to and including the striate cortex and suggest that the deficit shows a selectivity that is consistent with a loss to some localized prestriate or associated cortical area. This is in complete agreement with the CT scan results reported by Zihl et al. (1983) and those referred to here (see Materials and Methods).

\section{Properties of the deficit}

The main features of the motion deficit originally reported by Zihl et al. (1983) for this patient using more informal methods have been replicated in this study using visual stimuli of greater analytical power and psychophysical procedures that allow criterion-free estimates of performance. For velocities above about $10 \% \mathrm{sec}$ neither the direction of motion nor the relative velocity can be discriminated reliably. Even within the low-velocity range, performance for neither of these measures is normal. Sufficient investigation has been undertaken over the 2-3 years of this study to make us confident that these results are stable, not contaminated by practice effects and not due to a nonspecific attentional disturbance.

In an attempt to understand the nature of the visual deficit, we used 3 different but complementary approaches, namely, drift rate and temporal frequency discrimination, direction discrimination, and 2-flash apparent motion. The discrimination of drift rate of suprathreshold stimuli was particularly impaired, being only slightly better than that of a normal observer operating at detection threshold. For example, this patient could make only 3 discriminable steps across the entire temporal range for drifting grating stimuli and a suprathreshold stimulus drifting at $6 \% \mathrm{sec}$ could not be discriminated from any higher drift rate. Over no part of the range was discrimination normal. Interestingly, this rather dramatic loss was not solely restricted 


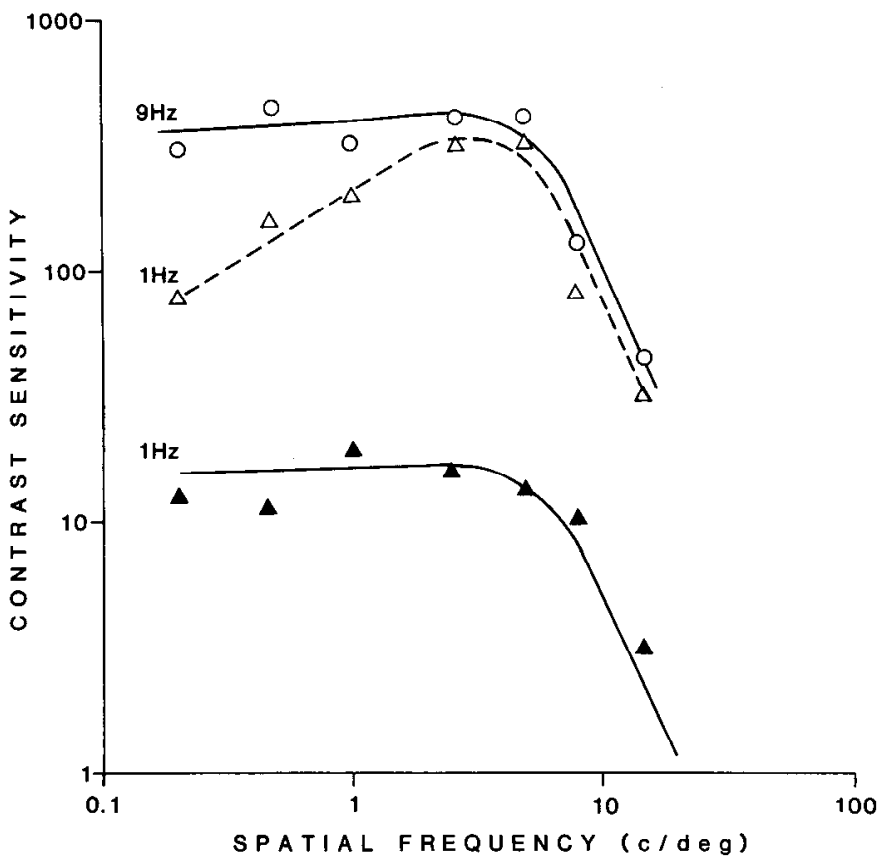

Figure 13. Results from Figure 12 replotted to show the spatial frequency dependence of the residual motion perception in this patient. Irrespective of the temporal frequency, it has a low-pass form and is displaced to lower absolute sensitivities.

to moving stimuli because a similar loss was demonstrated for contrast-reversing stimuli. Other spatial tasks such as spatial frequency and contrast discrimination were not affected to anywhere near the same extent. Thus, the overall deficit is specific to temporal processing, including motion, and is characterized by a large discrepancy between detection, which is relatively normal, and discrimination, which can be severely impaired.

The inability to perceive motion above about $6 \% \mathrm{sec}$ was substantiated by experiments in which the direction of motion was varied for stimuli at and about threshold. For drifting gratings of suprathreshold contrast, the direction of motion could not be reliably detected for velocities above $8 \%$ sec. For lower velocities, performance never reached normal limits for either stimulus. A clearer example of the difference between this patient's ability to detect the presence of moving stimuli and her ability to discriminate direction of motion is seen in the contrast sensitivity measurements for direction discrimination. In terms of sensitivity, her inability to perceive their motion is shown in the comparison of contrast thresholds for detection of the presence of drifting gratings, and contrast thresholds for the identification of their direction of drift. In sensitivity terms, there is about a factor of 10 between these 2 measures, and in terms of temporal acuity, about a factor of 5 . Furthermore, the residual motion perception is better described in terms of temporal frequency than it is in terms of velocity. The higher the temporal frequency, the more profound the contrast threshold elevation for directional discrimination. Thus, the overall results suggest that the local component information necessary for the derivation of motion is intact and that the anomaly occurs at a later stage, where a more global analysis takes place. In this way, it is similar to the distinction made by Zeki (1983) between wavelength-selective cells in striate cortex and color-coded cells in prestriate cortex and that of Movshon et al. (1983) between the component direction coding for cells in striate cortex as opposed to more global direction coding of compound (i.e., pattern direction of plaids) stimuli by cells in the prestriate cortex.

These findings bear an interesting resemblance to those in cats reared in a stroboscopically illuminated environment, in which the strobe frequency has been set to low enough $(8 \mathrm{~Hz})$ to preclude the "short-range" motion mechanism. Such cats have been demonstrated to have an almost complete loss of directionally selective neurons in striate cortex (Cynader and Chernenko, 1976; Pasternak et al., 1985), as well as in the lateral suprasylvian areas (Spear et al., 1985). Cats reared in this manner suffer a mild (2-fold) reduction of contrast sensitivity for detection of moving sinewave gratings but a much greater loss (10-fold) of sensitivily in discriminating the direction of motion of the same gratings (Pasternak and Leinen, 1986). Also, these cats exhibit decreased performance for temporal frequency discrimination of contrast-reversing gratings and a severe deficit for velocity discrimination of drifting gratings. It is not clear to what extent the visual anomalies of these animals should be attributed to loss of function in the striate cortex, as opposed to extrastriate areas (Spear et al., 1985). This comparison is considerably weakened by the known anatomical differences between the visual pathway in cat and primate, in particular the fact that extra striate areas in cat receive a substantial independent projection for the thalamus.

The 2-flash apparent motion experiments allow the spatial and temporal aspects of the deficit to be analyzed separately. These results suggest that the "short-range process" underlies the residual apparent motion perception in this patient. In addition to a deficit for short exposure durations not previously seen in the detection of individual spatiotemporal stimuli, there was also a reduction in the range of spatial displacements that support apparent motion, even for optimal conditions of ISI (e.g., short) and exposure duration (e.g., long). Even within this range, performance never reached normal limits. $D_{\max }$ was reduced by about a factor of 3 , and $D_{\min }$ increased by a factor of at least 3 .

\section{Explanations of the loss}

It is unlikely that the residual motion vision of this patient is derived secondarily from feature-tracking or relative displacement cues. While this could have been a possibility for wellstructured stimuli such as gratings, it is much more difficult to sustain this argument for random dots, especially when presented in 2-flash apparent motion. Another argument against such an interpretation is that similar defects were found for contrast-reversing stimuli.

Motion is presumably derived by comparing the outputs of arrays of spatiotemporal filters, and so one possible explanation is that the defect lies in this preprocessing stage. In terms of the visual pathway, this would correspond to neurons up to and including the striate cortex. Contrast sensitivity data for detection of sinewave gratings is generally believed to reflect the envelope of sensitivity of an array of tuned "channels." It is likely that these channels correspond to a population of neurons at a relatively low level of the visual pathway, probably striate cortex. The patient's relatively small deficit of contrast sensitivity for grating detection, and the dependence of these data on spatial and temporal frequency, suggests that this early stage of visual processing is largely intact. The conclusion based on 
psychophysical data is consistent with the available data on the patient's lesion from computerized axial tomography (see $\mathrm{Ma}$ terials and Methods), which implicate the superior temporal sulcus (and not occipital cortex). Since it is the comparison of filter outputs that is important to the derivation of motion, the abnormality must be located at or beyond this stage. Furthermore, since not all filter comparisons display comparable abnormalities (e.g., spatial and contrast), even in the presence of temporal modulation, one is led to conclude that the defect is selective for temporal filter comparisons, including those necessary for motion. This would suggest that the deficit is selective for only some prestriate processing. Since spatial acuity and color vision are normal (Zihl et al., 1983), and stereopsis is relatively normal, one would need to seek an explanation for this perceptual defect in some localized region beyond the striate cortex.

When speculating about the possible site of the underlying lesion from the properties of the residual vision, one must proceed with caution since it is known that there are multiple parallel visual projections. This means that one may need to consider 2 different types of explanation for the residual vision found in this patient. The first is that it represents the residual function of a normal motion pathway. The other is that is represents the rudimentary motion processing capabilities of a parallel pathway not primarily designed to handle motion but brought into play because of a complete interruption to the primary motion pathway.

Assuming that the first proposal is true, the results might suggest that the residual vision is mediated by area V5 (MT), known to be an important part of the primary motion pathway. Neurons in area V5 or MT have been shown to be responsible for directional responses to large spatial displacements for apparent motion and higher speeds for continuous motion (Zeki, 1971, 1974; Mikami et al., 1986a, b), and also to respond primarily to the velocity, rather than the temporal frequency, of grating stimuli (J. A. Movshon and W. T. Newsome, personal communication). Both the pattern of the spatial loss seen for apparent motion and the temporal frequency nature of the contrast threshold elevation for directional discrimination, together with the impaired discrimination of contrast-reversing stimuli, are inconsistent with this.

However, this does not mean that area V5 (MT) is not severely affected. Consider another type of explanation, namely, that the normal motion pathway has been totally interrupted by, for example, a lesion to area V5 (MT) and another parallel pathway is supporting the rudimentary motion perception that has been documented in this patient. Subcortically, directionally selective mechanisms that support optokinetic nystagmus may play a role in this patient's rudimentary motion perception. The other known parallel pathway is that of the parvocellular LGN/V1/V4 pathway, which is believed to carry chromatic and spatial information. This pathway is thought to carry only a rudimentary motion signal. For example, there is evidence that in normal vision only very rudimentary motion perception is possible using isoluminant color stimuli (Ramachandran and Gregory, 1975; Cavanagh et al., 1984; but also see Mullen and Baker, 1985). This patient's normal color discrimination, small but significant overall reduction in contrast sensitivity, the form of the spatial loss for apparent motion, and low-pass spatial properties for direction discrimination would all be consistent with such speculation.

\section{References}

Allman, J., F. Miezin, and E. L. McGuiness (1985) Direction- and velocity-specific responses from beyond the classical receptive field in the midde temporal visual area (MT). Perception 14: 105-126.

Andersen, R. A., and R. M. Siegel (1987) Effects of ibotenic acid lesions in area MT on motion perception thresholds in the Macaque monkey. Invest. Ophthalmol. Vis. Sci. Suppl. 28: 197.

Baker, C. L., Jr., and O. J. Braddick (1985) Eccentricity-dependent scaling of the limits fr short-range apparent motion perception. Vision Res. 25: 803-812.

Braddick, O. (1974) A short-range process in apparent motion. Vision Res. 14: 519-527.

Burr, D. C. (1979) On the visibility and appearance of objects in motion. Ph.D. dissertation, University of Cambridge, Cambridge.

Cavanagh, P., C. W. Tyler, and O. E. Favreau (1984) Perceived velocity of moving chromatic gratings. J. Opt. Soc. Am. A 1:893-899.

Cynader, M. S., and G. Chernenko (1976) Abolition of direction selectivity in the visual cortex of the cat. Science 193: 504-505.

Dubner, R., and S. M. Zeki (1971) Response properties and receptive fields of cells in an anatomically defined region of the superior temporal sulcus in the monkey. Brain Res. 35: 528-532.

Hess, R. F., and G. T. Plant (1985) Temporal frequency discrimination in human vision: Evidence for an additional mechanism in the low spatial and high temporal frequency region. Vision Res. 25: 14931500 .

Hess, R. F., and J. S. Pointer (1985) Differences in the neural basis of human amblyopia: The distribution of the anomaly across the visual field. Vision Res. 25: 1577-1594.

Mandler, M. B. (1984) Tempora frequency discrimination above threshold. Vision Res. 24: 1873-1880.

Maunsell, J. H. R., and D. C. van Essen (1983) Functional properties of neurons in middle temporal visual area of the macaque monkey. I. Selectivity for stimulus direction, speed and orientation. J. Neurophysiol. 49: 1127-1147.

Mikami, A., W. T. Newsome, and R. H. Wurtz (1986a) Motion selectivity in macaque visual corlex. I. Mechanisms in direction and speed selectivity in extrastriate area MT. J. Neurophysiol. 55: 13081327.

Mikami, A., W. T. Newsome, and R. H. Wurtz (1986b) Motion selectivity in macaque visual cortex. II. Spatiotemporal range of directional interactions in MT and V1. J. Neurophysiol. 55: 1328-1339.

Movshon, J. A., E. H. Adelson, M. S. Gizzi, and W. T. Newsome (1983) The analysis of moving visual patterns. In Pattern Recognition Mechanisms, C. Chagas, R. Gattass, \& C. Gross, eds., Pontificiae Academiae Scientiarum, Citta del Vatinano.

Mullen, K. T., and C. L. Baker (1985) A motion aftereffect from an isoluminant stimulus. Vision Res. 25: 685-688.

Newsome, W. T., R. H. Wurtz, M. R. Dursteler, and A. Mikami (1985) Deficits in visual motion processing following ibotenic acid lesions of the middle temporal visual area of the macaque monkey. J. Neurosci. 5: 825-840.

Newsome, W. T., A. Mikami, and R. H. Wurtz (1986) Motion selectivity in macaque visual cortex. III. Psychophysics and physiology of apparent motion. J. Neurophysiol. 55: 1340-1357.

Pastcrnak, T., and L. Lcinen (1986) Pattern and motion vision in cats with selective loss of cortical directional selectivity. J. Neurosci. 6 : 938-945.

Pasternak, T., R. A. Schumer, M. S. Gizzi, and J. A. Movshon (1985) Abolition of visual cortical direction selectivity affects visual behaviour in cats. Exp. Brain Res. $61: 214-217$.

Ramachandran, V. S., and R. L. Gregory (1975) Does colour provide an input to human motion perception. Nature $275: 55-56$.

Robson, J. G. (1966) Spatial and temporal contrast sensitivity functions of the visual system. J. Opt. Soc. Am. 56: 1141-1142.

Spear, P. D., L. Tong, M. A. McCall, and T. Pasternak (1985) Developmentally induced loss of direction selective neurones in the cat lateral suprasylvian visual cortex. Dev. Brain Res. 20: 281-285.

Tolhurst, D. J. (1975) Reaction times in the detection of gratings by human observers: A probabilistic mechanism. Vision Res. 15: 11431149.

Watson, A. B., and J. Nachmias (1977) Patterns of temporal interaction in the detection of gratings. Vision Res. 17: 893-902. 
Watson, A. B., and J. G. Robson (1981) Discrimination at threshold: Labelled detectors in human vision. Vision Res. 21: 1115-1122.

Zeki, S. M. (1971) Cortical projections from two prestriate areas in the monkey. Brain Res. 14: 19-35.

Zeki, S. M. (1974) Functional organization of a visual area in the posterior bank of the superior temporal sulcus of the rhesus monkey. J. Physiol. 236: 549-573.
Zeki, S. M. (1983) Colour coding in the cerebral cortex: The response of wavelength-selective and colour-coded cells in monkey visual cortex to changes in wavelength composition. Neuroscience 9:767-781.

Zihl, J., D. Von Cramon, and N. Mai (1983) Selective disturbance of movement vision after bilateral brain damage. Brain 106: 313-340. 